\title{
2-O-Methylmagnolol Induces Apoptosis and Inhibits IL-6/STAT3 Signaling in Oral Squamous Cell Carcinoma
}

\author{
Tong-Hong Wang ${ }^{a, b} \quad$ Jia-You Fang ${ }^{c, d} \quad$ Shu-Ju Wue Yi-Wen Liu $^{a} \quad$ Chieh-Wen Chan ${ }^{a}$ \\ Shih-Yi Chuang Chi-Yuan Chen ${ }^{a, b}$ \\ a'Graduate Institute of Health Industry Technology and Research Center for Industry of Human Ecology, \\ College of Human Ecology, Chang Gung University of Science and Technology, Tao-Yuan, 'Tissue Bank, \\ Chang Gung Memorial Hospital, Tao-Yuan, 'Pharmaceutics Laboratory, Graduate Institute of Natural \\ Products, Chang Gung University, Tao-Yuan, 'Department of Anesthesiology, Chang Gung Memorial \\ Hospital, Tao-Yuan, eDepartment of Nutrition and Health Sciences, Chang Gung University of Science \\ and Technology, Tao-Yuan, Taiwan
}

\section{Key Words}

Magnolol $\cdot$ MM1 $\cdot$ OSCC $・$ IL-6/STAT3 $\cdot$ Apoptosis

\begin{abstract}
Background/Aims: 2-O-methylmagnolol (MM1), a derivative of magnolol bearing one methoxy moiety, has been shown to display improved anti-tumor activity against skin cancers. In this study, we examined the anti-tumor effects of magnolol and MM1 on oral squamous cell carcinoma (OSCC). Methods: Trypane blue staining and clonogenic assays were performed to determine the cytotoxic effects of magnolol and MM1 in OSCC cells. Migration and matrigel invasion assays were carried out to examine the metastasis effects of magnolol and MM1 in OSCC cells. IL6-stimulation, Western blot, and immunohistochemistry were used to investigate the IL-6/STAT3 signaling and apoptosis. A bioluminescent mouse model of orthotopically implanted SAS cells was used to determine the anti-tumor activity of MM1 in vivo. Results: MM1 displays greater activity than magnolol on affecting the cytotoxicity, migration, and invasion of OSCC cells cultured in vitro. The improved anti-tumor activity of MM1 was shown to associate with its greater activity to inhibit STAT3 signaling and to induce apoptosis in the OSCC. In addition, we presented evidence that MM1 is effective in inhibiting the growth of orthotopic implanted OSCC cells in vivo. Conclusion: Our data indicate that MM1 displays greater anti-tumor activity than magnolol in OSCC and is an attractive agent to be further explored for its potential clinical application.




\section{Cellular Physiology Cell Physiol Biochem 2018;50:883-892

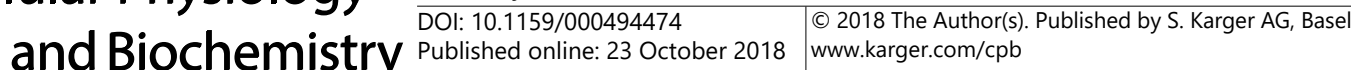

Wang et al.: 2-O-Methylmagnolol Induces Apoptosis in OSCC

\section{Introduction}

Oral squamous cell carcinoma (OSCC) is a malignancy of lip or oral cavity that commonly displays lymph node metastasis $[1,2]$. The incidence of this type of cancer is relatively high in South and Southeast Asia, including Taiwan [3]. The current treatment methods for OSCC patients include surgery, radiation therapy and chemotherapy [3]. However, the overall survival rate of treated OSCC patients remains unsatisfactory low [3]. A more effective treatment is desperately needed to improve the patient's survival.

Herbal medicine is the source of many currently available drugs [4]. Magnolia officinalis is a widely-used traditional Chinese medicine [5]. The bark of Magnolia is rich in a biphenol compound magnolol [5]. Magnolol has been displayed to induce apoptosis in the cells of many human cancers, including non-small cell lung cancer, breast cancer, bladder cancer, prostate cancer, gallbladder cancer, and colon cancer [6-12]. In addition, several studies have shown that magnolol also display anti-metastasis and anti-angiogenesis activity in ovarian, breast, and prostate cancer [13-15] Together, these studies have indicated that magnolol is a promising candidate for the derivation of more effective antitumor agents.

In an attempt to optimize the anti-inflammatory ability of magnolol, we have synthesized two methoxylated derivatives of magnolol, namely '2-Omethylmagnolol' (MM1) and 'dimethylmagnolol' (M2M) [16]. While both MM1 and M2M exhibited improved antiinflammatory activity [16], only MM1 displayed an increased anti-tumor activity against skin cancer cells than magnolol both in vitro and in vivo [17]. This finding suggests that MM1 may also display improved anti-tumor activity against other types of cancers, and therefore may be further explored as a potential therapeutic agent for many different cancers. In this study, we examined the antitumor activity of MM1 against OSCC cells cultured in vitro and in an orthotopic mouse model in vivo.

\section{Materials and Methods}

\section{Cell Culture}

SAS is a high-grade tumorigenic human tongue squamous cell carcinoma [18]. OECM1 is a nontumorigenic Taiwanese OSCC cell line. SAS and OECM1 cells were cultured in Dulbecco's modified Eagle's medium (DMEM) containing 10\% fetal bovine serum (FBS), $0.5 \mathrm{mM}$ sodium pyruvate, $1.2 \mathrm{~g} / \mathrm{L}$ sodium bicarbonate, and $2.5 \mathrm{mM}$ L-glutamine. Culture medium, chemical compounds, and FBS were purchased from Life Technologies (Grand Island, NY).

\section{Compounds, antibodies and plasmids}

Magnolol and 2-0-methylmagnolol (MM1) (Fig. 1A) were prepared as described by Lin et al. [16]. The purity of magnolol and MM1 was at least $99.5 \%$ as determined from HPLC analysis. Magnolol and MM1 were dissolved in dimethyl sulfoxide (DMSO) to make a stock concentration at $100 \mathrm{mM}$ and stored at $-20{ }^{\circ} \mathrm{C}$ before use. The $0.1 \% \mathrm{v} / \mathrm{v}$ DMSO was used as a vehicle control. Antibodies against phospho-STAT3 (Tyr705), STAT3, E-cadherin, vimentin, cleaved-PARP (cl-PARP, Asp214), caspase 8, caspase 9, and cleaved-caspase 3 (cl-caspase 3) were purchased from Cell Signaling (Temecula, CA, USA). The antibody against $\beta$-actin was purchased from Sigma (St. Louis, MO, USA). The plasmid pGL4.51 expressing the luciferase gene was purchased from Promega. Interleukin-6 (IL-6) was purchased from Cell Sciences (Canton, MA).

Assays for cell proliferation and clonogenic ability

Assays for viability (by staining with trypane blue), and clonogenic ability were performed as described previously $[17,19]$.

Invasion assay and migration assay

Invasion and migration assays were performed as described previously $[18,20]$. 


\section{Cellular Physiology Cell Physiol Biochem 2018;50:883-892 \begin{tabular}{ll|l} 
aOl: 10.1159/000494474 & $\begin{array}{l}\text { O 2018 The Author(s). Published by S. Karger AG, Basel } \\
\text { www.karger.com/cpb }\end{array}$
\end{tabular}}

Wang et al.: 2-O-Methylmagnolol Induces Apoptosis in OSCC

Western blotting

Western blotting was performed as described previously [18].

Construction of luciferase-expressing SAS cells

The luciferase-expressing SAS cells were constructed by transfection of pGL4.51 plasmid DNA into SAS cells using Lipofectamine 2000 (Invitrogen, Carlsbad, CA) [18]. After $24 \mathrm{~h}$, the transfected SAS cells were cultured in supplemented DMEM medium containing $1 \mathrm{mg} / \mathrm{ml} \mathrm{G418}$ for two weeks to obtain stably transfected SAS cells (SAS-Luc).

\section{In vitro bioluminescence imaging}

Serial dilutions of SAS-Luc cells ranging from $1 \times 10^{5}$ to $2 \times 10^{6}$ cells were seeded into each well of 96well culture plates. Luciferase assay reagent (Bright-Glo ${ }^{\mathrm{TM}}$ Luciferase Assay System; Promega) $100 \mu \mathrm{l}$ was added to the wells immediately before bioluminescence imaging. Wells containing medium only were used to detect background fluorescence. Photon counts per second were recorded using an IVIS100 (Xenogen, Alameda, CA) imaging system [21].

\section{A bioluminescent mouse model for orthotopically implanted OSCC}

The in vivo antitumor activity of MM1 against human OSCC was studied using male nude mice, BALB/c $\mathrm{nu} / \mathrm{nu}$ (supplied by the National Laboratory Animal Center, Taipei, Taiwan). The SAS-Luc cells $\left(3 \times 10^{5}\right)$ were injected into the tongue of nude mice. Bioluminescence imaging was done with an IVIS100 imaging system and performed as described previously [21]. The mice were randomly divided into groups of seven $(n=7)$ and drug treatment was commenced three days after tumor implantation, a time when the orthotopically implanted tumors could be detected by the bioluminescence imaging in all mice. Drug treatment was administered by intra-peritoneal (i.p.) injection of MM1 (5 mg per Kg of mice) daily. The control group received injection of DMSO. The growth of implanted tumors was monitored by the IVIS imaging. After 31 days, all mice were sacrificed by $\mathrm{CO}_{2}$ inhalation. The orthotopic tumors were excised, formalin fixed and paraffin embedded for immunohistochemistry (IHC) and routine hematoxylin and eosin (HE) staining. Animal experiments were performed under an approved protocol in accordance with the guidelines for the Animal Care Ethics Commission of Chang Gung Memorial Hospital (2016121301).

\section{Immunohistochemistry (IHC) and hematoxylin-eosin (HE) staining}

IHC and HE staining were performed as described previously [22]. The primary antibodies used for IHC staining were targeted against cleaved-caspase 3 (9546, Cell Signaling) or pSTAT3 (Tyr 705).

\section{Statistics}

Student's $t$-test was completed by the Statistical Package for the Social Sciences version 12.0 (SPSS, Inc.). Differences between the variables were considered significant for $p$ values less than 0.05 .

\section{Results}

MM1 displays increased cytotoxic activity than magnolol in OSCC cells cultured in vitro

The cytotoxic effects of MM1 and magnolol were examined in two OSCC cell lines, SAS and OECM1, using trypane blue staining method. As shown in Fig. 1B, MM1 displayed significantly increased cytotoxic activity than magnolol in both the SAS and OECM1 cells. The $\mathrm{IC}_{50}$ of these two drugs are summarized in Table 1. Consistent with the cytotoxic results, MM1 also displayed greater inhibition of clonogenic ability than magnolol in a highly malignant SAS cells (Fig. 1C). Together, these results showed that MM1 exhibited greater cytotoxic effect than magnolol in OSCC cells. 


MM1 displays
greater anti-
migration
and anti-
i $n$ v a s i o $n$
ability than
magnolol in
OSCC cells
$\mathrm{M}$ a g n o l o l

has been shown to display antimigration and invasion ability in breast and prostate cancers $[13,15]$. To evaluate whether or not MM1 may display greater anti-metastasis activity than $\mathrm{m}$ a $\mathrm{g} \mathrm{n}$ o l o l, we examined the effects of magnolol and MM1 on the migration and invasion abilities of OSCC cells. As shown in Fig. 2A, the motility of SAS and OECM1 cells treated with magnolol for 8 $\mathrm{h}$ or $16 \mathrm{~h}$ was not significantly reduced as compared to that of untreated cells. In contrast, the motility of SAS and OECM1 cells treated with MM1 for $16 \mathrm{~h}$ was significantly reduced as compared to that of untreated or magnolol-treated cells. After treatment of MM1 for $8 \mathrm{~h}$, only the motility of OECM1 cells was significantly reduced as compared to that of untreated cells. Similarly, MM1 also displayed significantly greater inhibition of invasion than magnolol in both SAS and OECM1 cells, as shown by a Matrigel invasion assay (Fig. 2B). Consistent with the greater ability of MM1 to inhibit invasion, we observed that the expression of vimentin, a mesenchymal marker, in MM1-treated cells was inhibited at a greater extent than that of magnolol-treated cells (Fig. 2C). Under the same experimental condition, the expression of E-cadherin, a cell-cell adhesion marker, was not significantly affected in both MM1-treated or magnololtreated cells.

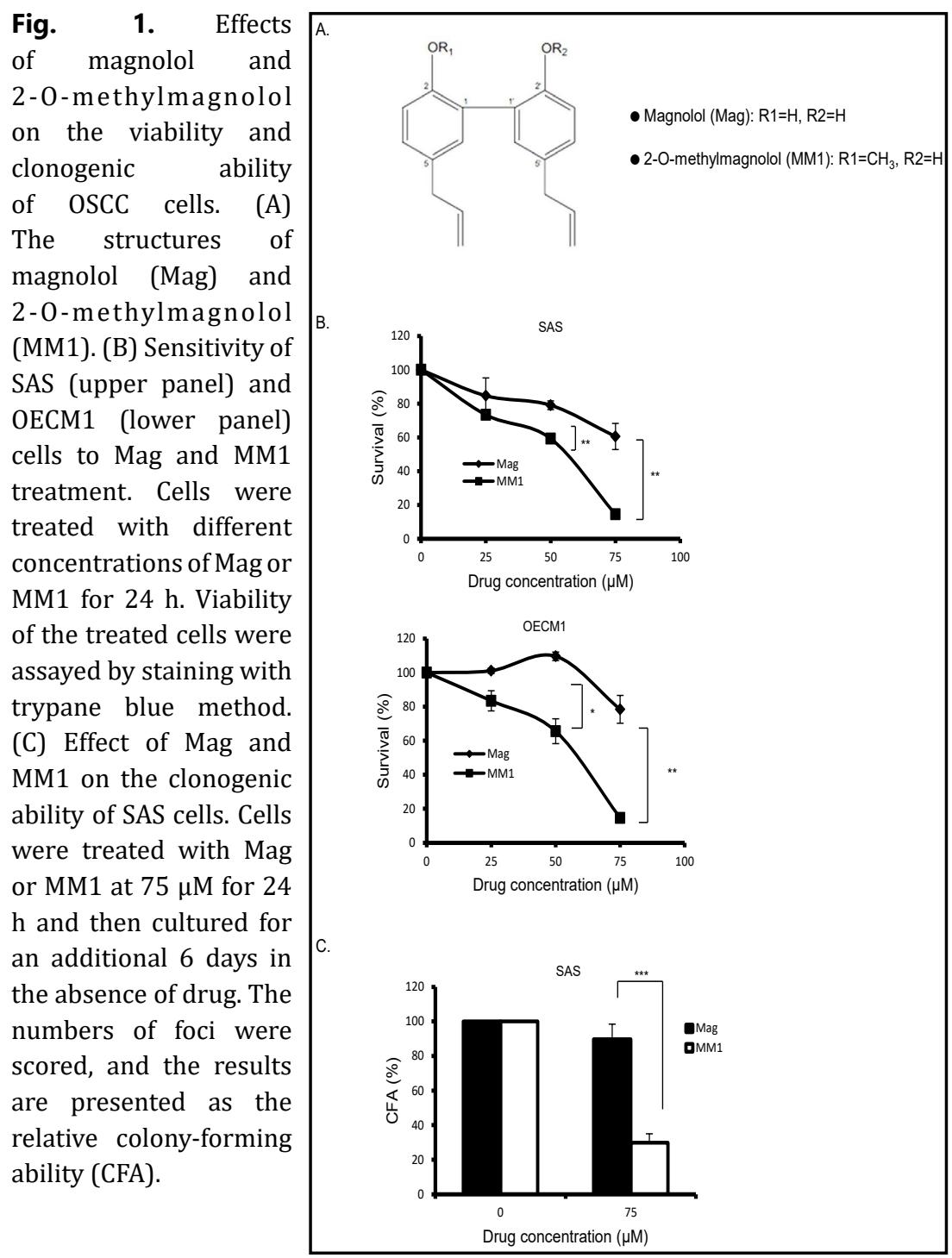

Table 1. The IC50 of magnolol and MM1 in human OSCC cells. ${ }^{a}$ Cells were treated with various concentrations of magnolol or MM1 for $24 \mathrm{~h}$ and the cell viability was determined by staining with trypane blue method. The IC50s were determined by nonlinear regression analysis. Values are given as means \pm standard deviation

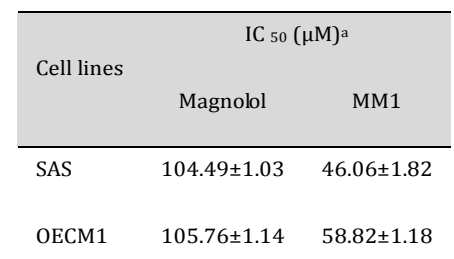




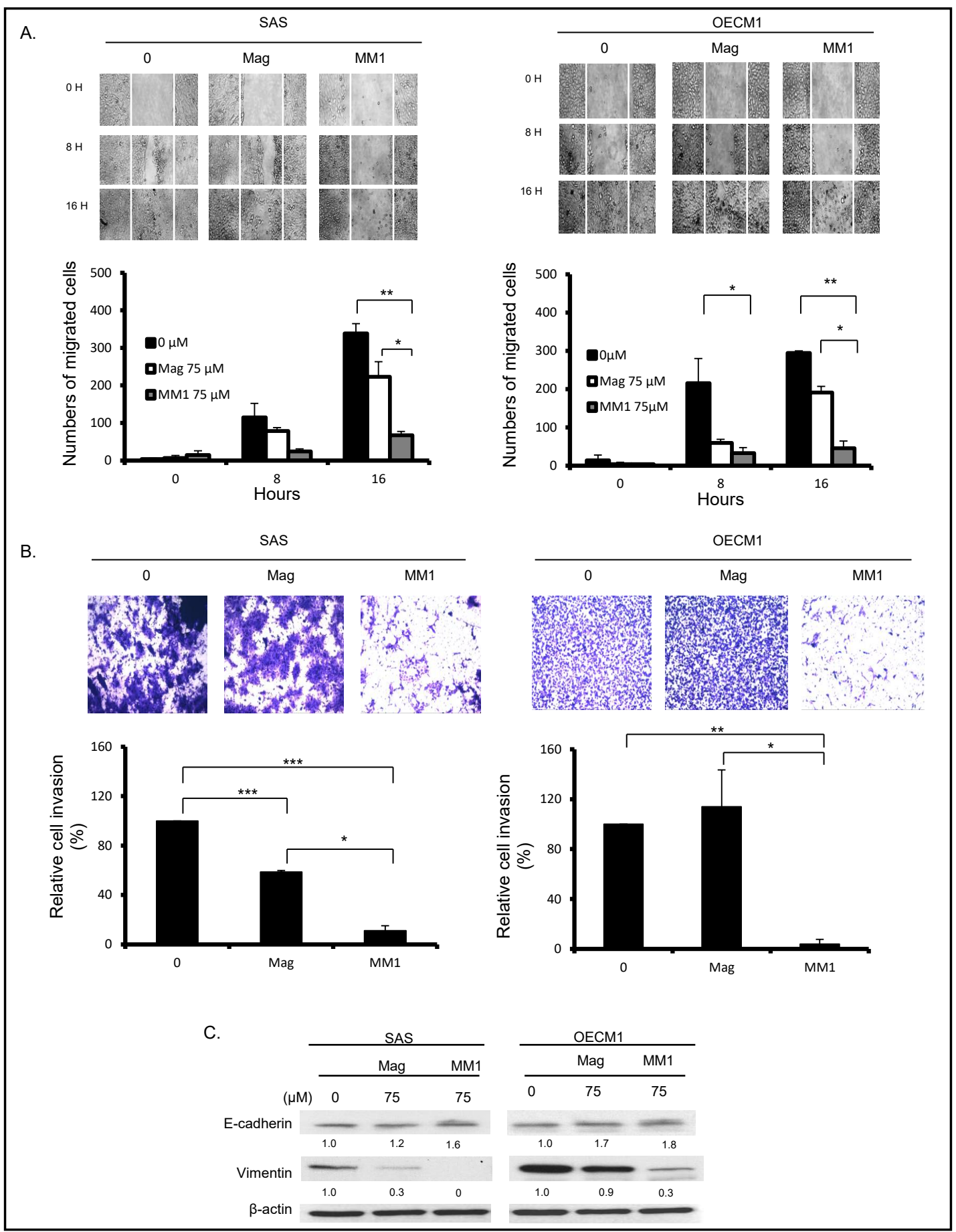

Fig. 2. Effects of Mag and MM1 on the migration and invasion ability of OSCC cells. SAS and OECM1 cells were treated Mag or MM1 at $75 \mu \mathrm{M}$, and the treated cells were assayed for: (A) Migration ability by wound healing assay, (B) Invasion ability by transwell chamber assay, and (C) Expression of E-cadherin and vimentin by Western blot analysis (C) as described in the Materials and Methods. Data are expressed as the mean \pm SD of three independent experiments. Symbols: ${ }^{*} \mathrm{p}<0.05 ;{ }^{* *} \mathrm{p}<0.01$; and ${ }^{* * *} \mathrm{p}<0.001$, as analyzed by unpaired $\mathrm{t}$-tests. In the Western blot analysis for E-cadherin and vimentin (C), $\beta$-actin was used as a loading control. The data presented are from one of three experiments with similar results. 


\section{\begin{tabular}{lll} 
Cellular Physiology & \multicolumn{1}{c}{ Cell Physiol Biochem 2018;50:883-892 } \\
DOl: 10.1159/000494474 & O 2018 The Author(s). Published by S. Karger AG, Basel
\end{tabular} \begin{tabular}{ll|l} 
and Biochemistry & $\begin{array}{l}\text { DOI: 10.1159/000494474 } \\
\text { Published online: } 23 \text { October } 2018\end{array}$ & $\begin{array}{l}\text { (c) } 2018 \text { The Author(s). Published by S. Karger AG, Basel } \\
\text { www.karger.com/cpb }\end{array}$ \\
\hline
\end{tabular}}

Effects of magnolol and MM1 on apoptosis and IL6-STAT3 pathway in OSCC cells

The increased antitumor activity of MM1 has been shown to correlate with its increased ability to induce apoptosis in skin cancer cells [17]. In the same study, we have presented preliminary data to indicate that such a mechanism also apply to oral cancer cells SAS. To further confirm this postulate, we examined the effects of magnolol and MM1 to induce apoptosis in OECM1 cells. As shown in Fig. 3A, the cleavages of caspase-3, caspase-8, caspase-9, and PARP were readily detected in the cells treated with MM1, but not with magnolol. These results indicate that MM1 also displays a greater ability than magnolol to induce apoptosis in OSCC cells.

It has been suggested that the improved antitumor activity of MM1 in skin cancer cells may attribute to the upregulation of IncRNA GAS5 and the enhancement of apoptosis [17]. While we have observed that MM1 displays a greater ability than magnolol to induce apoptosis in OSCC cells (Fig. 3A) [17], however, we were not able to detect any upregulation of IncRNA GAS5 in MM1treated OSCC cells (data not shown). To explore the potential mechanism for the enhanced apoptosis by MM1, we examined the effects of magnolol and MM1 on the STAT3-mediated signaling, since the STAT3-mediated signaling has been shown to be involved in OSCC proliferation, tumorigenesis and metastasis [23, 24], and is known to be suppressed by magnolol [25, 26]. As shown in Fig. 3B, the levels of endogenous pSTAT3 (Tyr705) were reduced more in SAS and OECM1 cells treated with MM1 more than those treated with magnolol. To confirm that MM1 has greater inhibitory ability on STAT3-mediated signaling, we examined the effects of magnolol and MM1 on IL-6 stimulated STAT3signaling. Cells were starved of serum for $24 \mathrm{~h}$, incubated with magnolol or MM1 at 25-75 $\mu$ M or DMSO for $1 \mathrm{~h}$, and then treated with $10 \mathrm{ng} / \mathrm{ml} \mathrm{IL-6}$ for

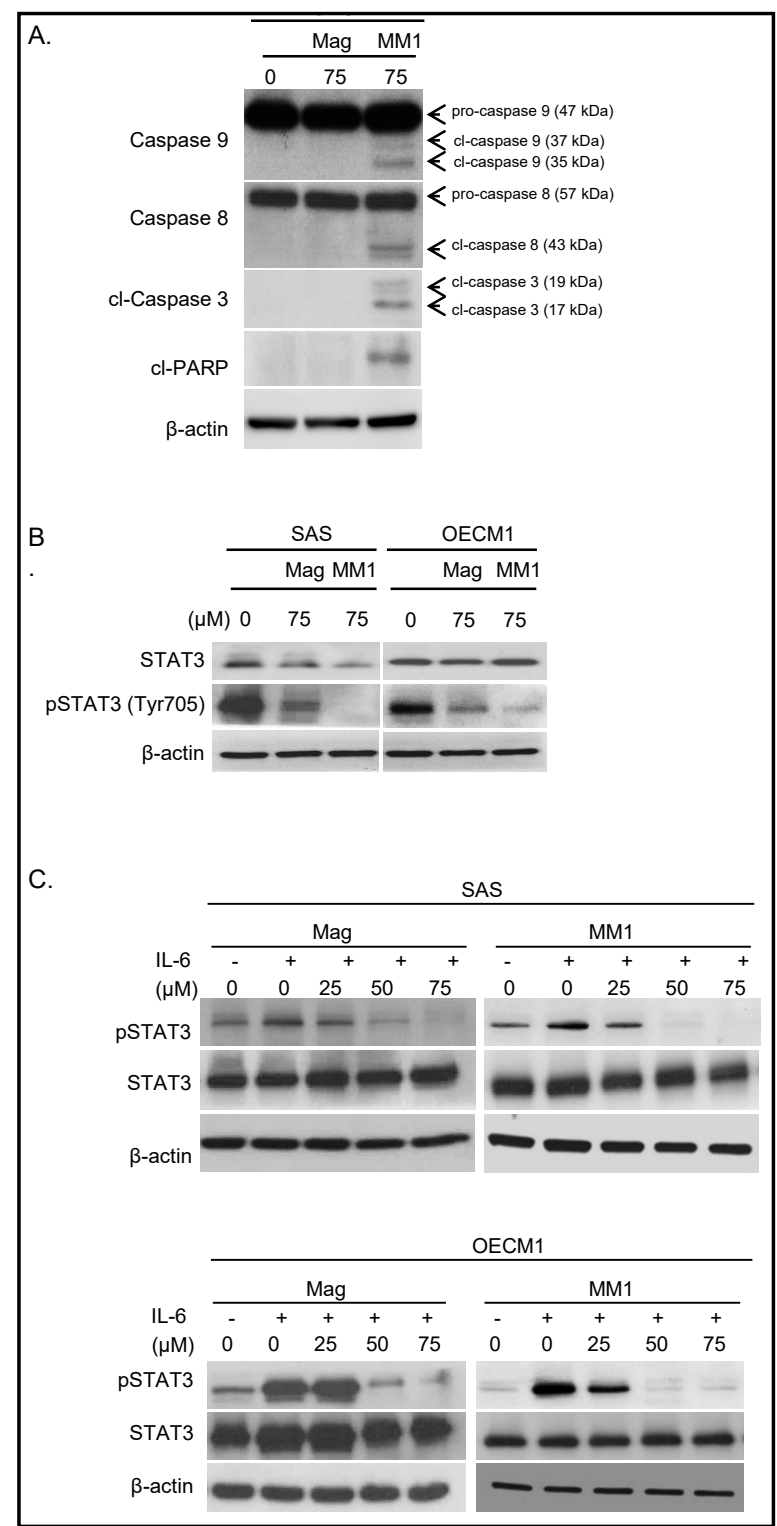

Fig. 3. Effect of Mag and MM1 on apoptosis and STAT3 signaling in OSCC cells. (A) OECM1 cells were treated with Mag or MM1 at $75 \mu \mathrm{M}$ for $24 \mathrm{~h}$, and the cell lysates of treated cells were analyzed for the cleavage of PARP, procaspase- $9,-8$, and -3 by Western blotting. (B) SAS and OECM1 cells were treated with Mag or MM1 at $75 \mu \mathrm{M}$ for $24 \mathrm{~h}$ and the cell lysates of treated cells were assayed for STAT3 and phosphorylated STAT3 (pSTAT3) by Western blotting. (C) SAS (upper panel) and OECM1 (lower panel) cells were cultured in serum-free medium for $24 \mathrm{~h}$, treated with various concentrations of Mag or MM1 for $1 \mathrm{~h}$, and then exposed to IL-6 at $10 \mathrm{ng} / \mathrm{ml}$ for $20 \mathrm{~min}$ in the presence of drug. The cell lysate were assayed for STAT3 and phosphorylated STAT3 (pSTAT3) by Western blotting. $\beta$-actin was used as a loading control in the Western blot analysis. These data presented are from one of three experiments with similar results. 


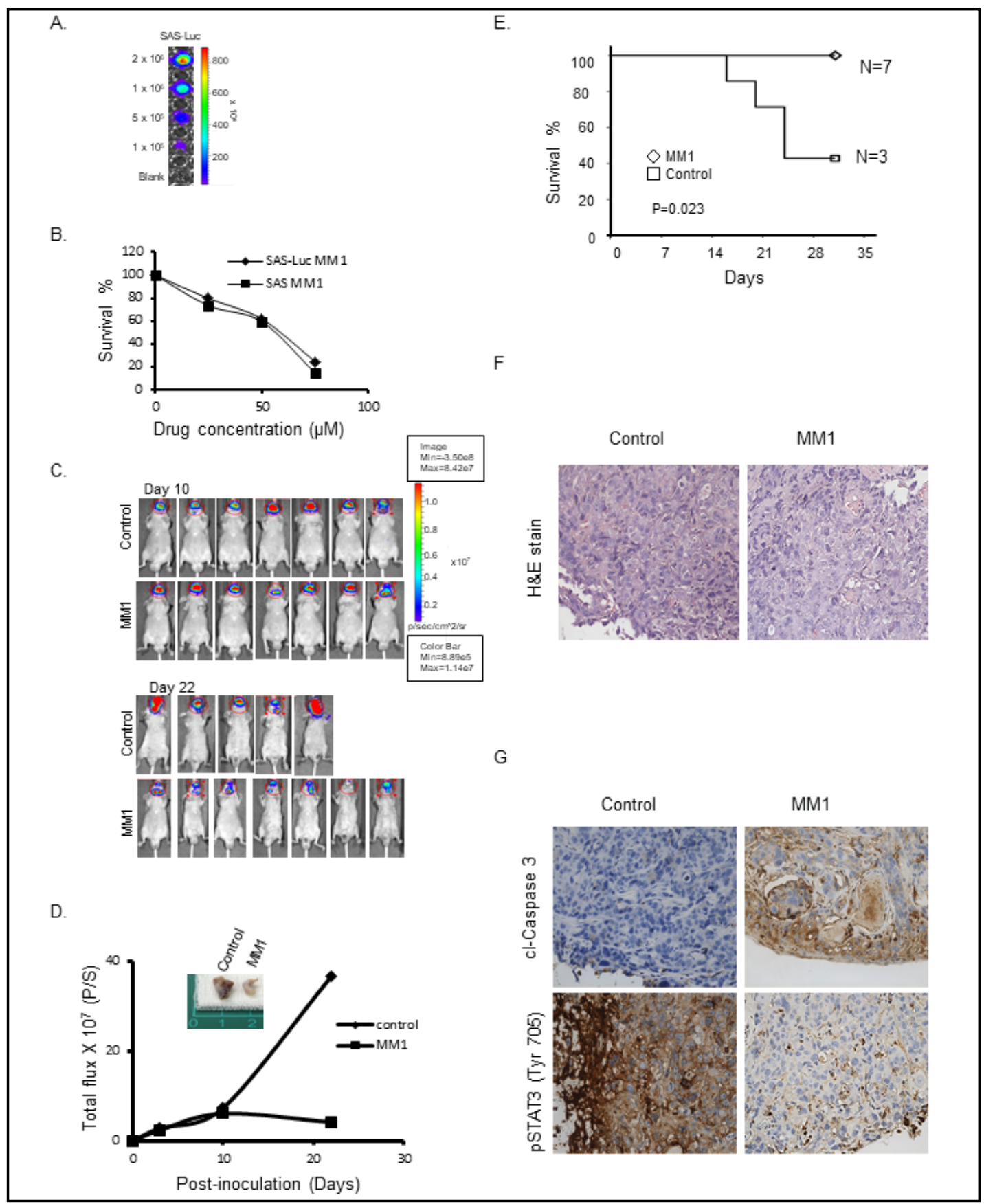

Fig. 4. The antitumor activity of MM1 against OSCC in a bioluminescent orthotopic implanted mouse model. (A) The expression of luciferase activity was monitored by bioluminescence imaging of SAS-Luc cells using an IVIS100 imaging system. (B) Sensitivity of SAS and SAS-Luc cells to MM1 treatment. SAS and SAS-Luc cells were treated with different concentrations of MM1 for $24 \mathrm{~h}$. Viability of the treated cells were assayed by staining with trypane blue method. (C-G) The in vivo antitumor activity of MM1 on the orthotopic implanted OSCC tumors. The SAS-Luc cells were inoculated into the tongue of nude mice. After 3 days of SAS-Luc cells inoculation, MM1 treatment (5 mg per Kg of mice) was administered intraperitoneally every day. Prior to IVIS imaging, the mice were injected intraperitoneally with $150 \mathrm{mg}$ D-luciferin/Kg of mice, and immediately imaged with sequential 30 seconds exposures. Representative IVIS images of control and MM1-treated mice are shown in (C). The total flux values of control and MM1-treated mice are shown in (D). The K-M survival of control and MM1-treated mice are shown in (E). Representative hematoxylin-eosin (HE) and IHC staining of excised tumors are shown in (F) and $(G)$, respectively. 
20 min. As shown in Fig. 3 C, MM1 displayed a greater inhibition of IL6-stimulated STAT3 signaling than magnolol in SAS and OECM1 cells.

\section{Antitumor effects of MM1 in vivo}

The in vivo antitumor activities of MM1 were evaluated using a bioluminescent mice model of orthotopic implanted SAS cells. The luciferase-expressing SAS (SAS-Luc) cells retain the same sensitivity to MM1 as the parental SAS cells and their relative numbers could be evaluated by the expressed luciferase activity (Fig. 4 A and B). To evaluate the antitumor activity of MM1 in vivo, the SAS-Luc cells were implanted into the tongue of nude mice. Mice were randomly separated into control and MM1 groups after 3 days of implantation, because the bioluminescence of orthotopic tumors could be readily detected in all mice at this time. MM1 treatment ( $5 \mathrm{mg}$ per $\mathrm{Kg}$ of mice) was performed by intraperitoneal injection daily and the growth of implanted tumors was monitored by the IVIS imaging (once per week) until day 31. As shown in Fig. 4C and 4D, the progression of orthotopic tumors was not significantly suppressed in the MM1-treated mice for the first 10 days, but was greatly suppressed at later times in the MM1-treated mice at day 22. Importantly, the survival of untreated mice from the control-group was reduced to about $43 \%$ at day 31 , while all of the MM1-treated mice remained viable (Fig. 4E). Hematoxylin and eosin staining of orthotopic tumors from the control-group displayed higher cell density than that from the MM1treated tumor (Fig. 4F). Finally, immunohistochemistry staining for cleavage caspase 3 (clcaspase 3) and phosphorylation STAT3 at Tyr705 revealed that the MM1-treated tumors has enhanced staining of cl-caspase 3, but reduced staining of pSTAT3 at Tyr705, which are consistent with results of Western blot analysis in the cultured OSCC cells (Fig. 3). Together, these results indicate that the MM1 suppressed the OSCC progression by inducing apoptosis and inhibiting STAT3 activation in vivo.

\section{Discussion}

Numerous signaling pathways have been implicated in the regulation of apoptosis by magnolol [27]. Magnolol triggers the release of Bid, Bax and cytochrome $\mathrm{c}$ from mitochondria, and induces apoptosis via a caspase-independent pathway in non-small cell lung cancer cells [7]. Induction of apoptosis by magnolol has been reported to mediate through caspaseindependent pathway and G2/M phase arrest in human breast cancer cells [9], and through cytochrome c/caspase 3/PARP and PTEN/Akt pathways in thyroid carcinoma cells [28]. The regulatory pathways of MM1-induced apoptosis in different cancers, on the other hand, are poorly understood at present.

In this study, we have shown that MM1 displays greater cytotoxic activity against OSCC cells cultured in vitro as compared to that of magnolol (Fig. 1). The greater cytotoxic activity of MM1 against OSCC cells appears to attribute, at least in part, to its greater activity to induce apoptosis (Fig. 3A), which is similar to that observed for the improved antitumor activity of MM1 against skin cancer cells [17]. However, the molecular basis for the improved antitumor activity of MM1 against these two types of cancer cells may not be the same. While we have previously reported that the improved antitumor activity of MM1 against skin cancer cells is associated with its greater activity to up-regulate the expression of lncRNA GAS5 and to induce apoptosis [17], we were not able to detect increased expressions of IncRNA GAS5 in MM1-treated OSCC cells. Instead, MM1 appeared to display a greater ability than magnolol to inhibit STAT3 signaling (Fig. 3), the major driver pathway in OSCC proliferation, tumogenesis and metastasis $[23,24]$. Therefore, we are inclined to conclude that the improved anti-tumor activity of MM1 is attributed to its greater activity to inhibit STAT3 signaling in OSCC cells.

We have previously shown that MM1 displays antitumor activity against xenograft skin tumors in nude mice [17]. As the results from present work indicate that MM1 also displayed greater antitumor activity than magnolol against cultured OSCC cells, we chose to only examine the in vivo antitumor effects of MM1 on OSCC cells to minimize the use of animals. 


\section{Cellular Physiology Cell Physiol Biochem 2018;50:883-892

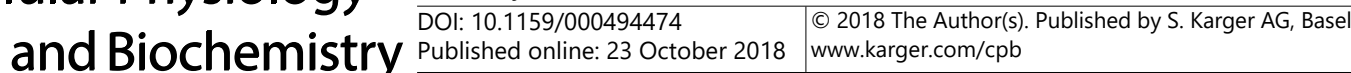

Wang et al.: 2-O-Methylmagnolol Induces Apoptosis in OSCC

Here, we presented evidence that MM1 is also an effective antitumor agent against OSCC in vivo (Fig. 4). Consistent with the findings from our in vitro studies, the antitumor activity of MM1 in vivo is shown to associate with its activity to inhibit STAT3 signaling and to induce apoptosis in the orthotopic implanted SAS cells (Fig. 4G).

High vimentin expression is known to associate with the STAT3 activation and metastatic risk of head and neck squamous cell carcinoma patients, including OSCC [29-31]. In this study, we have shown that MM1 also displayed greater ability to suppress invasion, migration, and vimentin expression than magnolol in OSCC cells (Fig. 2). Therefore, it is anticipated that MM1 could bear improved anti-metastasis activity than magnolol in OSCC. Further in vivo studies are needed to test the validity of this postulate.

\section{Acknowledgements}

We thank Dr. Tzu-Chien V. Wang for valuable suggestions and editorial assistance. This work was supported by grants from Chang Gung Memorial Hospital (CMRPF1E0023), Ministry of Science and Technology of Taiwan (105-2320-B-255-001- and 105-2320-B-182A-004-). The funders had no role in study design, data collection, data analysis, publication decisions or manuscript preparation.

\section{Disclosure Statement}

The authors state that they have no conflicts of interest.

\section{References}

1 Thompson L: World Health Organization classification of tumours: pathology and genetics of head and neck tumours. Ear Nose Throat J 2006;85:74.

2 Chi AC, Day TA, Neville BW: Oral cavity and oropharyngeal squamous cell carcinoma--an update. CA Cancer J Clin 2015;65:401-421.

3 Rivera C: Essentials of oral cancer. Int J Clin Exp Pathol 2015;8:11884-11894.

4 Katiyar SK: Emerging Phytochemicals for the Prevention and Treatment of Head and Neck Cancer. Molecules 2016;21:pii: E1610.

5 Lee YJ, Lee YM, Lee CK, Jung JK, Han SB, Hong JT: Therapeutic applications of compounds in the Magnolia family. Pharmacol Ther 2011;130:157-176.

6 Li M, Zhang F, Wang X, Wu X, Zhang B, Zhang N, Wu W, Wang Z, Weng H, Liu S, Gao G, Mu J, Shu Y, Bao R, Cao Y, Lu J, Gu J, Zhu J, Liu Y: Magnolol inhibits growth of gallbladder cancer cells through the p53 pathway. Cancer Sci 2015;106:1341-1350.

7 Tsai JR, Chong IW, Chen YH, Hwang JJ, Yin WH, Chen HL, Chou SH, Chiu CC, Liu PL: Magnolol induces apoptosis via caspase-independent pathways in non-small cell lung cancer cells. Arch Pharm Res 2014;37:548-557.

-8 McKeown BT, McDougall L, Catalli A, Hurta RA: Magnolol causes alterations in the cell cycle in androgen insensitive human prostate cancer cells in vitro by affecting expression of key cell cycle regulatory proteins. Nutr Cancer 2014;66:1154-1164.

-9 Zhou Y, Bi Y, Yang C, Yang J, Jiang Y, Meng F, Yu B, Khan M, Ma T, Yang H: Magnolol induces apoptosis in MCF7 human breast cancer cells through G2/M phase arrest and caspase-independent pathway. Pharmazie 2013;68:755-762.

10 Chen MC, Lee CF, Huang WH, Chou TC: Magnolol suppresses hypoxia-induced angiogenesis via inhibition of HIF-1alpha/VEGF signaling pathway in human bladder cancer cells. Biochem Pharmacol 2013;85:12781287. 


\section{Cellular Physiology Cell Physiol Biochem 2018;50:883-892 \begin{tabular}{c|c|c|c|} 
DOI: 10.1159/000494474 & O 2018 The Author(s). Published by S. Karger AG, Basel \\
www.karger.com/cpb
\end{tabular}}

and Biochemistry $\frac{\text { Published onl }}{\text { Wang et al:: 2-O-Methylmagnolol Induces Apoptosis in OSCC }}$

11 Park JB, Lee MS, Cha EY, Lee JS, Sul JY, Song IS, Kim JY: Magnolol-induced apoptosis in HCT-116 colon cancer cells is associated with the AMP-activated protein kinase signaling pathway. Biol Pharm Bull 2012;35:16141620.

12 Kang YJ, Park HJ, Chung HJ, Min HY, Park EJ, Lee MA, Shin Y, Lee SK: Wnt/beta-catenin signaling mediates the antitumor activity of magnolol in colorectal cancer cells. Mol Pharmacol 2012;82:168-177.

13 Liu Y, Cao W, Zhang B, Liu YQ, Wang ZY, Wu YP, Yu XJ, Zhang XD, Ming PH, Zhou GB, Huang L: The natural compound magnolol inhibits invasion and exhibits potential in human breast cancer therapy. Sci Rep 2013;3:3098.

14 Chuang TC, Hsu SC, Cheng YT, Shao WS, Wu K, Fang GS, Ou CC, Wang V: Magnolol down-regulates HER2 gene expression, leading to inhibition of HER2-mediated metastatic potential in ovarian cancer cells. Cancer Lett 2011;311:11-19.

15 Hwang ES, Park KK: Magnolol suppresses metastasis via inhibition of invasion, migration, and matrix metalloproteinase-2/-9 activities in PC-3 human prostate carcinoma cells. Biosci Biotechnol Biochem 2010;74:961-967.

16 Lin CF, Hwang TL, Al-Suwayeh SA, Huang YL, Hung YY, Fang JY: Maximizing dermal targeting and minimizing transdermal penetration by magnolol/honokiol methoxylation. Int J Pharm 2013;445:153-162.

17 Wang TH, Chan CW, Fang JY, Shih YM, Liu YW, Wang TV, Chen CY: 2-O-Methylmagnolol upregulates the long non-coding RNA, GAS5, and enhances apoptosis in skin cancer cells. Cell Death Dis 2017;8:e2638.

18 Chen CY, Chiou SH, Huang CY, Jan CI, Lin SC, Hu WY, Chou SH, Liu CJ, Lo JF: Tid1 functions as a tumour suppressor in head and neck squamous cell carcinoma. J Pathol 2009;219:347-355.

19 Yeh YM, Chen CY, Huang PR, Hsu CW, Wu CC, Wang TC: Proteomic analyses of genes regulated by heterogeneous nuclear ribonucleoproteins A/B in Jurkat cells. Proteomics 2014;14:1357-1366.

20 Chen CY, Yang SC, Lee KH, Yang X, Wei LY, Chow LP, Wang TC, Hong TM, Lin JC, Kuan C, Yang PC: The antitumor agent PBT-1 directly targets HSP90 and hnRNP A2/B1 and inhibits lung adenocarcinoma growth and metastasis. J Med Chem 2014;57:677-685.

-21 Chen CY, Chang PC, Wang TH, Wang TV: The in vivo anti-leukemia activity of N-(1-Pyrenlyl) maleimide in a bioluminescent mouse model. Leuk Res 2017;62:64-69.

22 Chen CY, Jan CI, Lo JF, Yang SC, Chang YL, Pan SH, Wang WL, Hong TM, Yang PC: Tid1-L Inhibits EGFR Signaling in Lung Adenocarcinoma by Enhancing EGFR Ubiquitinylation and Degradation. Cancer Res 2013;73:4009-4019.

23 Sen M, Johnston PA, Pollock NI, DeGrave K, Joyce SC, Freilino ML, Hua Y, Camarco DP, Close DA, Huryn DM, Wipf P, Grandis JR: Mechanism of action of selective inhibitors of IL- 6 induced STAT3 pathway in head and neck cancer cell lines. J Chem Biol 2017;10:129-141.

24 Yadav A, Kumar B, Datta J, Teknos TN, Kumar P: IL-6 promotes head and neck tumor metastasis by inducing epithelial-mesenchymal transition via the JAK-STAT3-SNAIL signaling pathway. Mol Cancer Res 2011;9:1658-1667.

25 Chilampalli C, Guillermo R, Zhang X, Kaushik RS, Young A, Zeman D, Hildreth MB, Fahmy H, Dwivedi C: Effects of magnolol on UVB-induced skin cancer development in mice and its possible mechanism of action. BMC Cancer 2011;11:456.

-26 Chen SC, Chang YL, Wang DL, Cheng JJ: Herbal remedy magnolol suppresses IL-6-induced STAT3 activation and gene expression in endothelial cells. Br J Pharmacol 2006;148:226-232.

27 Xu HL, Tang W, Du GH, Kokudo N: Targeting apoptosis pathways in cancer with magnolol and honokiol, bioactive constituents of the bark of Magnolia officinalis. Drug Discov Ther 2012;5:202-210.

28 Huang SH, Chen Y, Tung PY, Wu JC, Chen KH, Wu JM, Wang SM: Mechanisms for the magnolol-induced cell death of CGTH W-2 thyroid carcinoma cells. J Cell Biochem 2007;101:1011-1022.

29 Nijkamp MM, Span PN, Hoogsteen IJ, van der Kogel AJ, Kaanders JH, Bussink J: Expression of E-cadherin and vimentin correlates with metastasis formation in head and neck squamous cell carcinoma patients. Radiother Oncol 2011;99:344-348.

-30 Zhou J, Tao D, Xu Q, Gao Z, Tang D: Expression of E-cadherin and vimentin in oral squamous cell carcinoma. Int J Clin Exp Pathol 2015;8:3150-3154.

31 Mali SB: Review of STAT3 (Signal Transducers and Activators of Transcription) in head and neck cancer. Oral Oncol 2015;51:565-569. 